\title{
Gouty arthritis and kidney function outcomes and serum uric acid level variations in obese patients following bariatric surgery
}

\author{
Mona G. Balata', Ahmed H. Helal ${ }^{2}$, AshrafH. Mohamed ${ }^{3 *}$, Alaa-Uddin Habib ${ }^{3}$, Mahmoud Awad ${ }^{3}$ and \\ Mohamed Sherif ${ }^{3}$
}

\begin{abstract}
Background: Obesity is an independent risk factor for chronic kidney disease (CKD) and is the strongest known modifiable risk factor for hyperuricemia and gout. We aimed to discover the outcome of serum uric acid (SUA), gouty arthritis, and kidney function in obese patients after bariatric surgery and possible links with BMI variations.

Methods: Retrospective study has been performed in National Hospital in Riyadh, KSA, between Jan. 2018 to Jan. 2020. We studied only 98 patients who met our inclusion criteria. Patients followed-up at 1 month (for gouty attack only) postoperative, 3 months postoperative, and 6 months postoperative for body mass index (BMI), serum creatinine, dipstick urinalysis, SUA, and estimated glomerular filtration rate (eGFR). Radiological studies, medical history, follow up radiological studies, and clinical follow up were obtained from the hospital data system.

Results: A total of 98 patients with mean eGFR were $90.65 \pm 29.34 \mathrm{ml} / \mathrm{min} / 1.73 \mathrm{~m}^{2}$, mean SUA $5.56 \pm 1.84 \mathrm{mg} / \mathrm{dl}$, and mean BMI was $45.28 \pm 7.25 \mathrm{~kg} / \mathrm{m}^{2}$, at surgery. Mean BMI had decreased significantly to $38.52 \pm 6.05 \mathrm{~kg} / \mathrm{m}^{2}$ at 3 months and to $34.61 \pm 5.35 \mathrm{~kg} / \mathrm{m}^{2}$ at 6 months $(P<0.001)$. The mean GFR had improved significantly $(99.14 \pm 23.32$ $\left.\mathrm{ml} / \mathrm{min} / 1.73 \mathrm{~m}^{2}\right)$ at 6 months $(P<0.001)$. Interestingly, proteinuria had resolved in 17 patients out of 23 patients at 6 months. Number of gouty attacks was decreased during the first month post-surgery $(P<0.001)$. SUA level was significantly decreased $(4.32 \pm 1.27 \mathrm{mg} / \mathrm{dl})(P<0.001)$. SUA showed significant negative correlations with eGFR at 3 months and positively significant correlations with $\mathrm{BMI}$ at 3 and 6 months. By multinomial logistic regression, $\mathrm{BMI}$ and initial eGFR were the independent predictive variables for the outcome of eGFR at 6 months, while male gender and initial SUA were the independent predictive variables on the outcome of SUA at 6 months. Postoperatively in gouty arthritis patients, the number of joints affected, patient global VAS assessment, and number of gouty attacks were significantly reduced $(P<0.001)$.
\end{abstract}

Conclusion: Bariatric surgery has been associated with reduction of BMI and subsequently reduction of SUA levels, gouty attacks, and improvement of eGFR.

Keywords: Bariatric, BMI, Gout, Uric acid, GFR

\footnotetext{
*Correspondence: mashraf2011@yahoo.com

${ }^{3}$ Department of Internal Medicine, Faculty of Medicine, Mansoura University, Mansoura, Egypt

Full list of author information is available at the end of the article
}

\begin{abstract}
Introduction
Obesity is one of the most common preventable diseases [1], with multifactorial etiology [2]. Bariatric surgery has been considered the most reliable method of achieving long-term weight loss [3]. Bariatric surgery is associated with a significant decrease in body weight and serum uric acid (SUA) levels among people with obesity,
\end{abstract}


with predominantly obvious reductions in those with hyperuricemia and gout [3]. However, few studies have reported on the longitudinal changes in uric acid levels after bariatric surgery [4]. Obesity is an independent risk factor for hyperuricemia and gout, particularly visceral adiposity, via both increased production and decreased renal excretion of uric acid [5]. CKD is an evolving noncommunicable disease of public health significance [6]. Obesity is well-defined as an independent risk factor for CKD incidence and its progression [7]. Morbid obesity is linked to glomerulosclerosis [8]. Renal diseases in the setting of obesity often manifest by proteinuria, glomerular hyperfiltration, and decreased glomerular filtration rate (GFR) [9]. Numerous studies have shown an association between obesity and both the development and the progression of CKD and extends to other complications such as nephrolithiasis and kidney malignancies $[10,11]$.

We aimed to discover the outcome of SUA, gouty arthritis, and kidney function in obese patients after bariatric surgery and in correlation with BMI variations.

\section{Methods}

\section{Study design}

Retrospective study had been performed in National Hospital in Riyadh, KSA, between Jan. 2018 to Jan. 2020. All the preoperative assessment and the postoperative follow-up data were collected from the hospital data base system. We studied 166 obese patients requiring surgical treatment for the first time (72 men, 94 women); only 98 patients (41 males and 57 females) were included and met our inclusion criteria. Biochemical parameters were done by obtaining blood samples after an overnight fast, included blood urea nitrogen (BUN), serum creatinine, serum uric acid (SUA), and eGFR by using modification diet for renal disease study equation (DMRD).

For gouty patients (35 patients), medication history and clinical examination (radiological follow-up if available) from the hospital system were collected; it was included, disease duration, number of joint affected, visual analog scale (VAS) patient global assessment (0-100 score) [12], number of tophi seen in affected joints, and number of gouty attack before and 6 months after the operation.

\section{Inclusion and exclusion criteria}

Eligibility criteria included:

(I)Age ranges from 18 to 65 years old

(II)BMI more than 40 or more than 35 with associated high-risk family history of DM, and cardiovascular disease for which nonsurgical treatment failed

(III)Any patient with history of DM, hypertension, chronic kidney disease, and any chronic disease was excluded from the study
(IV)History of steroid therapy and/or thyroid dysfunction were excluded

(V)Patients with history of previous bariatric surgery, debilitating disease, unresolved psychiatric illness, unable to follow-up, smoking, or substance abuse were excluded from the study

\section{Follow-up}

Patients followed-up at 1-month (for gouty attack only) postoperative, 3-month postoperative, and 6-month postoperative for BMI, serum creatinine, dipstick urine analysis for proteinuria, serum uric acid and eGFR, and clinical follow-up for gouty patients.

\section{Statistical analysis}

All data will be analyzed using the SPSS statistical software for Windows, version 21.0, data were expressed as means \pm SD (number and percent for categorical data). Comparisons of continuous data were performed using an independent sample $t$ test/pair $t$ test or the MannWhitney $U$ test where appropriate while categorical data were compared using chi-square test. Correlations between BMI and uric acid, arthritis outcome and eGFR were identified by using partial correlation analysis. Multinomial logistic regression analysis was performed to assess the independent predictive effects of the variables on variance of SUA and eGFR. $P$ value less than 0.05 was considered significance.

\section{Results \\ Clinical characteristics \\ At the surgery}

Of the 98 patients, $57(58.2 \% \%)$ were female, the mean age was $33.28 \pm 11.38$ years, the mean BMI was 45.65 $\pm 7.25 \mathrm{~kg} / \mathrm{m}^{2}$, the mean SUA was $5.56 \pm 1.84 \mathrm{mg} / \mathrm{dl}$, and the eGFR (using MDRD) was $90.65 \pm 29.34 \mathrm{~mL} /$ $\min / 1.73 \mathrm{~m}^{2}$. Twenty-three patients $(23.5 \%)$ had significant proteinuria ( $\geq 0.5 \mathrm{~g} /$ day) and $12(12.24 \%)$ patients with history of recent gouty attack (Table 1).

\section{At 3 months post-surgery}

There was significant reduction in BMI, SUA, number of patient with proteinuria, and number of gouty attacks, and eGFR compared to the starting values $(P<0.001)$ (Table 1).

\section{At 6 months post-surgery}

There was significant reduction in BMI, SUA, number of patient with proteinuria and number of gouty attacks, and marked improvement of eGFR compared to the starting and after 3 months values $(P<0.001)$ (Table 1$)$. 
Table 1 Demographic and laboratory data of the studied group at the surgery, after 3 months, and after 6 months

\begin{tabular}{|c|c|c|c|c|c|}
\hline & At the start & After 3 months & $P$ value & After 6 months & $P$ value \\
\hline Gender (M/F) (n) & $41 / 57$ & $41 / 57$ & & $41 / 57$ & $N S^{*}$ \\
\hline Age (years) & $33.28 \pm 11.38$ & $33.52 \pm 11.38$ & NS & $33.77 \pm 11.38$ & NS \\
\hline BWT (kg) & $124.80 \pm 23.94$ & $106.07 \pm 20.15$ & $<0.001$ & $95.33 \pm 18.13$ & $<0.001$ \\
\hline $\mathrm{BMI}\left(\mathrm{kg} / \mathrm{m}^{2}\right)$ & $45.28 \pm 7.25$ & $38.52 \pm 6.05$ & $<0.001$ & $34.61 \pm 5.35$ & $<0.001$ \\
\hline Creatinine (mg/dl) & $0.89 \pm 0.21$ & $0.85 \pm 0.16$ & $<0.001$ & $0.79 \pm 0.14$ & $<0.001$ \\
\hline Proteinuria no. & $75 / 23$ & $85 / 14$ & $<0.001^{*}$ & $92 / 6$ & $<0.001$ \\
\hline Proteinuria (mg/day) & $117.43 \pm 12.55$ & $106.51 \pm 8.96$ & $<0.001$ & $101.66 \pm 4.84$ & $<0.001$ \\
\hline Serum UA (mg/dl) & $5.56 \pm 1.84$ & $4.43 \pm 1.22$ & $<0.001$ & $4.32 \pm 1.27$ & $<0.001$ \\
\hline Abn.UA (N/H) & $59 / 39$ & $72 / 26$ & $<0.001^{*}$ & $90 / 8$ & $<0.001^{*}$ \\
\hline Gouty attacks & $12 / 88$ & $5 / 93$ & $<0.001^{*}$ & $2 / 96$ & $<0.001^{*}$ \\
\hline eGFRm (ml/min) & $90.65 \pm 29.34$ & $>90 \pm 24.01$ & $<0.001$ & $>90 \pm 23.32$ & $<0.001$ \\
\hline
\end{tabular}

$M$ males, $F$ females, $B M I$ body mass index, $k g$ kilogram, $m^{2}$ meter square, $m g$ milligram, $d l$ deciliter, - negative, + positive, $U A$ uric acid, $N$ normal serum level, $H$ high serum level, eGFRc estimated glomerular filtration rate by Cockcroft-Gault formula, eGFRm estimated glomerular filtration rate by modification diet for renal disease study equation (DMRD)

*Chi-square test

Table 2 eGFR outcome in the studied group

\begin{tabular}{llll}
\hline Items & Low eGFR & Normal eGFR & High eGFR \\
\hline At the surgery & 51 & 42 & 5 \\
After 3 months & 45 & 51 & 3 \\
After 6 months & 35 & 60 & 3 \\
$P$ value & $<0.001$ & $<0.001$ & NS \\
\hline
\end{tabular}

eGFR and serum uric acid variance during the study period Of 98 patients, at the surgery, 51 (52\%) patients had low eGFR (MDRD), 42 (42.86\%) patients had normal eGFR and only 5 (5.1\%) patients had abnormal high eGFR. After 3 month, 45 (45.9\%) patients had low eGFR (MDRD), 51 (52\%) had normal eGFR and only 3 (3.06\%) patients had abnormal high eGFR $(P<0.001)$. After 6 month, 35 (35.7\%) patients had low eGFR (MDRD), 60 (61.22\%) had normal eGFR, and only 3 (3.06\%) patients had abnormal high eGFR. There was significant improvement (normalization) $(P<0.001)$ of abnormal lower eGFR after 6 month post-surgery (Table 2). SUA level was decreased significantly during the study period $(P<0.001)$ and was correlated and linked to reduction in BMI and improvement in eGFR (Tables 1, 3, and 5).

\section{Correlations}

\section{At the start and 3 months after surgery}

SUA showed significant negative correlations with eGFR (MDRD) and significant positive correlations with BMI (Table 3).

\section{At 6 months post-surgery}

SUA showed significant positive correlations to BMI (Table 3) and no correlation with eGFR (MDRD) (Table 3). For eGFR (Cockcroft-Gault formula), there were positive correlations with serum creatinine and BMI and no any significant correlation with SUA.

Table 3 Correlation between eGFRm, BMI, and serum UA in the studied group (Spearman correlation)

\begin{tabular}{|c|c|c|c|c|c|c|}
\hline \multirow[t]{2}{*}{ Parameters } & \multicolumn{2}{|c|}{ Serum UAO } & \multicolumn{2}{|c|}{ Serum UA3 } & \multicolumn{2}{|c|}{ Serum UA6 } \\
\hline & $r$ & $P$ value & $r$ & $P$ value & $r$ & $P$ value \\
\hline eGFR 0 & -.200 & 0.048 & & & & \\
\hline eGFR 3 & & & -.221 & 0.029 & & \\
\hline eGFR 6 & & & & & NS & \\
\hline BMI 0 & .206 & 0.042 & & & & \\
\hline BMI 3 & & & .221 & 0.029 & & \\
\hline BMI 6 & & & & & .218 & 0.033 \\
\hline
\end{tabular}

UAO uric acid at surgery, $U A 3$ uric acid at 3 months post-surgery, UA6 uric acid at 6 months post-surgery 
Table 4 Multinomial logistic regression for independent predictive variable on eGFR outcome

\begin{tabular}{|c|c|c|c|c|c|c|c|c|}
\hline \multirow[t]{2}{*}{ Parameters } & \multirow[t]{2}{*}{$B$} & \multirow[t]{2}{*}{ Std. error } & \multirow[t]{2}{*}{ Wald } & \multirow[t]{2}{*}{$d f$} & \multirow[t]{2}{*}{ Sig. } & \multirow[t]{2}{*}{$\operatorname{Exp}(B)$} & \multicolumn{2}{|c|}{$95 \% \mathrm{Cl}$ for exp. $(B)$} \\
\hline & & & & & & & Lower bound & Upper bound \\
\hline Age & .088 & .056 & 2.515 & 1 & .113 & 1.092 & .979 & 1.218 \\
\hline BWT & -.056 & .034 & 2.682 & 1 & .101 & .945 & .884 & 1.011 \\
\hline BMI & -.276 & .120 & 5.314 & 1 & .021 & 1.318 & 1.042 & 1.666 \\
\hline S. creatinine & 3.168 & 4.260 & .553 & 1 & .457 & 23.769 & .006 & 1.004 \\
\hline SUA & -.195 & .209 & .873 & 1 & .350 & .823 & .546 & 1.239 \\
\hline eGFR (starting) & .190 & .054 & 12.382 & 1 & .000 & 1.209 & 1.088 & 1.344 \\
\hline Proteinuria & .773 & .945 & .493 & 1 & .483 & .504 & .250 & 18.742 \\
\hline
\end{tabular}

Table 5 Multinomial logistic regression for independent predictive variable on SUA outcome

\begin{tabular}{|c|c|c|c|c|c|c|c|c|}
\hline \multirow[t]{2}{*}{ Parameters } & \multirow[t]{2}{*}{$B$} & \multirow[t]{2}{*}{ Std. error } & \multirow[t]{2}{*}{ Wald } & \multirow[t]{2}{*}{$d f$} & \multirow[t]{2}{*}{ Sig. } & \multirow[t]{2}{*}{$\operatorname{Exp}(B)$} & \multicolumn{2}{|c|}{$95 \% \mathrm{Cl}$ for exp. (B) } \\
\hline & & & & & & & Lower bound & Upper bound \\
\hline Age & .481 & 1.432 & .133 & 1 & .737 & 1.617 & .098 & 26.718 \\
\hline BWT & -.039 & .044 & .799 & 1 & .371 & .962 & .883 & 1.048 \\
\hline BMI & -.199 & .171 & 1.346 & 1 & .246 & .820 & .586 & 1.147 \\
\hline Creatinine & -9.678 & -9.678 & 8.715 & 1 & .267 & 6.268 & 2.493 & 16.412 \\
\hline SUA & -2.014 & 1.024 & 3.871 & 1 & .049 & .133 & .018 & .992 \\
\hline eGFR & -.066 & .067 & .971 & 1 & .324 & .936 & .820 & 1.068 \\
\hline Gender (males) & 6.256 & 6.256 & 3.138 & 1 & .046 & 5.209 & 1.110 & 24.447 \\
\hline
\end{tabular}

\section{Multinomial logistic regression}

We found that only BMI and initial eGFR were the independent predictive variables on the outcome of eGFR at 6 months $(P=0.021$ and $<0.001$, respectively) (Table 4) and male gender and initial uric acid were the independent predictive variables on the outcome of SUA after 6 months $(P=0.046$ and 0.049 , respectively) (Table 5).

\section{Gouty arthritis patient group}

The demographic, laboratory data, and clinical parameters were concised in Table 6; there were significant reduction in BMI, SUA, and serum creatinine, number of joints affected postoperative, VAS assessment postoperative, and number of gouty attacks postoperatively compared with preoperative state $(P<0.001)$ while there was significant improvement of eGFR and disappearance of joint tophi at 6 months postoperative. At 6 months postoperative, BMI reduction was positively correlated with SUA $(P<0.001)$ and with patient global assessment VAS score $(P=0.045)$, while postoperative gouty attack reduction was positively correlated with patient global VAS $(P=0.003)$ and with postoperative number of joint affected $(P=0.013)$. There was on noticed tophi deposition around the previously affected joints.
Table 6 Parameters gouty arthritis patient group

\begin{tabular}{llll}
\hline Parameters & Preoperative & $\begin{array}{l}\mathbf{6} \text { months } \\
\text { postoperative } \\
\text { Mean } \pm \text { SD }\end{array}$ & P value \\
\hline Age & $34.80 \pm 12.64$ & $35.33 \pm 12.64$ & NS \\
Disease duration & $5.14 \pm 3.48$ & $5.58 \pm 3.48$ & NS \\
BMl & $46.82 \pm 9.00$ & $35.47 \pm 6.52$ & $<0.001$ \\
Serum creatinine & $0.96 \pm 0.20$ & $0.82 \pm 0.14$ & $<0.001$ \\
Serum uric acid & $5.67 \pm 1.91$ & $4.69 \pm 1.55$ & $<0.001$ \\
eGFR & $79.77 \pm 22.81$ & $93.14 \pm 19.13$ & $<0.001$ \\
No. of joints affected & $2.14 \pm 0.88$ & $0.54 \pm 0.66$ & $<0.001$ \\
Presence of tophi & $0.11 \pm 0.32$ & 0.00 & $<0.001$ \\
Patient global VAS & $5.23 \pm 2.26$ & $1.49 \pm 1.12$ & $<0.001$ \\
score & & & $0.14 \pm 0.36$ \\
No. of gouty attacks & $1.83 \pm 0.79$ & & \\
\hline
\end{tabular}

\section{Discussion}

Bariatric surgery is associated with a major improvement in dyslipidemia, hypertension, urinary albumin excretion, quality of life, and with an important improvement in all parameter of kidney function [13-15]. Glomerulomegaly and focal glomerulosclerosis have been closely linked to obesity and may lead to initiation of glomerular hyperfiltration and deterioration of kidney functions [16]. 
In our study, a total of 98 patients with mean GFR $90.65 \mathrm{ml} / \mathrm{min} / 1.73 \mathrm{~m}^{2}$, mean SUA $5.56 \mathrm{mg} / \mathrm{dl}$, and mean BMI $45.28 \mathrm{~kg} / \mathrm{m}^{2}$ before surgery. BMI had significantly decreased to $38.52 \mathrm{~kg} / \mathrm{m}^{2}$ at 3 months and to $34.61 \mathrm{~kg} /$ $\mathrm{m}^{2}$ at 6 months $(P<0.001)$. The mean eGFR at 3 and 6 months of follow-up had improved significantly to $>90$ $(P<0.001)$. Interestingly, proteinuria had resolved significantly in 17 patients out of 23 patients after 6 months $(P$ $<0.001$ ) (Table 1). Many studies showed that glomerular hyperfiltration caused by obesity reflected loss of renal functional reserve and shared to the progressive of CKD $[17,18]$. Many studies confirm a beneficial relationship of bariatric surgery with long-term kidney function course using multiple filtration markers [19]. The significant association between bariatric surgery and improved kidney function trajectory in patients at lower baseline kidney function was consistent with other studies that only examined eGFRcr [20], as in our study, there was significant improvement of lowered eGFR followed to bariatric surgery $(P<0.001)$ (Table 2$)$. Patient who failed to recover their eGFR, it was less than $60 \mathrm{~mL} / \mathrm{min} / 1.73 \mathrm{~m}^{2}$. By multinomial logistic regression, we found that only BMI and initial eGFR were the independent predictive variables on the outcome of eGFR at 6 months $(P=0.021$ and $<0.001$, respectively) (Table 4 ). It was clear that bariatric surgery obviously improved all major risk factors for kidney injury as well as parameters of kidney function (GFR, urinary protein excretion, but also may be linked to a major increase in risk of stone formation) [21]. Using of multiple filtration equation to show homogeneity of the association with improved kidney functions is a good, but we found that MDRD was more reliable than Cockcroft-Gault formula in obese patients. Education and awareness of the risks of obesity and a healthy lifestyle, including proper nutrition and exercise, can intensely help in preventing obesity and kidney disease [22].

Abdominal adiposity and obesity were linked to high risk of increasing gout [23]. Bariatric surgery has been related to decreased SUA levels and overall gout incidence. Weight loss showed beneficial effects on SUA and gout attacks $[24,25]$. Increased SUA seems to be related to decreased eGFR [26, 27]. Our work showed that SUA had significantly decreased to $4.43 \pm 1.22 \mathrm{mg} /$ $\mathrm{dl}$ at 3 months and to and $4.32 \pm 1.27 \mathrm{mg} / \mathrm{dl}$ at 6 months postoperative (Table 1) which were in line with a study reported that the incidence of SUA levels were decreased just 1 month postoperatively [28]. Although Dalbeth et al. [29] reported an increase in SUA 2 weeks after surgery and they suggested that it was due to renal dysfunction associated with major surgery, or metabolic effects from fasting or rapid weight loss (catabolic state), our work found that postoperative in gouty arthritis patients, the number of joints affected, VAS global assessment score, and number of gouty attacks were significantly reduced $(P<0.001)$ (Table 6$)$ which was in line with other study [28] which linked this outcome to decreased BMI, SUA, and improved GFR. We reported that BMI reduction was positively correlated with SUA $(P<0.001)$ and with VAS global assessment score $(P=0.045)$, while postoperative gouty attack reduction was positively correlated with VAS $(P=0.003)$ and with postoperative number of joint affected $(P=0.013)$ (Table 6). There is a higher risk of gout attacks during the first month postoperative [30] mainly related to the changes in SUA, rather than absolute level [11] as dramatic changes in SUA levels might trigger gout attacks [31] as crystals may be shed back to the bloodstream and lead to the time-based increase of SUA and trigger acute gouty attack and the flare-up [3]. On the other hand, weight loss following bariatric surgery was found to be associated with reduced inflammatory responses to monosodium urate (MSU) crystals and significant reductions in many inflammatory cytokines [32] leading to significant reduction in the frequency of gout attacks [33, 34]. Others reported that the effect of bariatric surgery on SUA levels and the frequency of gout attacks vary in time after the surgery $[28,35]$. Gout may be another indication for bariatric surgery in patients with obesity [3]. There is a relationship between SUA and gender. Males with a BMI over $27.5 \mathrm{~kg} / \mathrm{m}^{2}$ were 16 times more likely to have gout attacks than males with a BMI $<20 \mathrm{~kg} / \mathrm{m}^{2}$ [25]. In our study, males showed a greater decline in SUA than females and males had high mean SUA $8.15 \pm 0.63 \mathrm{mg} / \mathrm{dl}$ while females have $7.17 \pm 0.65$ $\mathrm{mg} / \mathrm{dl}$ at the start, but at 6 months postoperative; males had mean SUA $5.09 \pm 1.40 \mathrm{mg} / \mathrm{dl}$ while females have mean SUA $5.18 \pm 1.31 \mathrm{mg} / \mathrm{dl}$. Males showed significant reduction in SUA by $93.33 \% 6$ months after surgery, while in females showed $56.57 \%$ reduction $(p<0.001)$. By multinomial logistic regression, we found that male gender and initial uric acid were the independent predictive variables on the outcome of uric acid at 6 months $(P=0.046$ and 0.049 , respectively) (Table 5). Our results were supported by many studies $[28,36]$ and partially with others [37]. The study had weak points and limitations, being a retrospective study and short period of follow-up.

\section{Conclusion}

Bariatric surgery has a great and significant effect on patient outcome: marked improvement of eGFR and excellent reduction of SUA and improvement of gouty arthritis among obese patients.

\section{Acknowledgements \\ Not applicable}

\section{Authors' contributions}

M. S. Sharing idea of the study, data analysis, writing discussion and publication. M.G. Sharing idea of the study, data collection and processing, result 
and discussion writing. A. H. Sharing idea of the study, data analysis, writing discussion and publication. A. H. Sharing idea of the study, data collection and processing, result and discussion writing. M. A. Sharing idea of the study, data analysis, writing discussion and publication. A. M. Sharing idea of the study, data collection and processing, data analysis, result and discussion writing. All authors read, reviewed, and approved the manuscript.

\section{Funding}

Not applicable

\section{Availability of data and materials}

The data that support the finding of this study are available from the National Hospital in Riyadh, KSA, but restriction apply to the availability of the data which were used under license in the current study and local license from that hospital and so are not publicly available. Data are however available from the corresponding author upon reasonable request and with permission from the National Hospital.

\section{Declarations}

\section{Consent to participate}

Not applicable (retrospective study)

\section{Ethics approval and consent to participate}

The study was approved by the scientific and ethical committee in National Hospital, Riyadh, on February 2021.

\section{Competing interests}

The authors declare that they have no competing interests.

\section{Author details}

${ }^{1}$ Physical Medicine, Rheumatology, and Rehabilitation Department, Faculty of Medicine, Ain Shams University, Cairo, Egypt. ${ }^{2}$ Department of Internal Medicine, Faculty of Medicine, Ain Shams University, Cairo, Egypt. ${ }^{3}$ Department of Internal Medicine, Faculty of Medicine, Mansoura University, Mansoura, Egypt.

Received: 3 September 2021 Accepted: 2 December 2021

Published online: 10 January 2022

\section{References}

1. Baetge C, Earnest CP, Lockard B et al (2017) Efficacy of a randomized trial examining commercial weight loss programs and exercise on metabolic syndrome in overweight and obese women. Appl Physiol Nutr Metab 42(2):216-227.

2. Jukaku SA, Williams SRP (2021) The cause of obesity is multifactorial but GPs can do more. BMJ 373:n956. https://doi.org/10.1136/bmj.n95.

3. Lu J, Bai Z, Chen Y et al (2021) Effects of bariatric surgery on serum uric acid in people with obesity with or without hyperuricaemia and gout: a retrospective analysis. Rheumatology. https://doi.org/10.1093/rheum atology/keaa822

4. Sjostrom L, Lindroos AK, Peltonen M et al (2004) Lifestyle, diabetes, and cardiovascular risk factors 10 years after bariatric surgery. N Engl J Med 351:2683-2693

5. Rathmann W, Funkhouser E, Dyer AR et al (1998) Relations of hyperuricaemia with the various components of the insulin resistance syndrome in young black and white adults: the CARDIA study. Coronary artery risk development in young adults. Ann Epidemiol 8(4):250-261

6. Rai PK, Jindal PK, Rai P et al (2014) Screening of chronic kidney disease (CKD) in general population on world kidney day on three consecutive years: a single day data. Int J Med Public Health 4:167-170

7. Kovesdy CP, Furth SL, Zoccali C, World Kidney Day Steering Committee (2017) Obesity and kidney disease: hidden consequences of the epidemic. Nephron 135:243-251

8. Kambham N, Markowitz GS, Valeri AM, Lin J et al (2001) Obesity-related glomerulopathy: an emerging epidemic. Kidney Int 59:1498-1509

9. Serra A, Romero R, Lopez D et al (2008) Renal injury in the extremely obese patients with normal renal function. Kidney Int 73:947-955
10. Brolin RE (2002) Bariatric surgery and long-term control of morbid obesity. JAMA 288:2793-2796

11. Mingrone G, Panunzi S, De Gaetano A et al (2012) Bariatric surgery versus conventional medical therapy for type 2 diabetes. N Engl J Med 366(17):1577-1585

12. Hawker GA, Mian S, Kendzerska T et al (2011) Measures of adult pain visual analog scale for pain (VAS pain), numeric rating scale for pain (NRS pain), McGill pain questionnaire (MPQ), short-form McGill pain questionnaire (SF-MPQ), chronic pain grade scale (CPGS), short form-36 bodily pain scale (SF-36 BPS), and measure of intermittent and constant osteoarthritis pain (ICOAP). Arthritis Care Res 63(S11):S240-SS25

13. Navaneethan SD, Kelly KR, Sabbagh F et al (2010) Urinary albumin excretion, HMWadiponectin, and insulin sensitivity in type 2 diabetic patients undergoing bariatric surgery. Obes Surg 20(3):308-315. https://doi.org/ 10.1007/s11695-009-0026-1.

14. Habib P, Scrocco JD, Terek M et al (2009) Effects of bariatric surgery on inflammatory, functional and structural markers of coronary atherosclerosis. Am J Cardiol 104:1251-1255

15. Ahmed MH, Byrne CD (2010) Bariatric surgery and renal function: a precarious balance between benefit and harm. Nephrol Dial Transplant 25:3142-3147

16. Li K, Zou J, Ye Z et al (2016) Effects of bariatric surgery on renal function in obese patients: a systematic review and meta-analysis. PLOS ONE 11(10):e0163907.

17. Helal I, Fick-Brosnahan GM, Reed-Gitomer B, Schrier RW (2012) Glomerular hyperfiltration: definitions, mechanisms and clinical implications. Nat Rev Nephrol 8:293-300

18. Chagnac A, Herman M, Zingerman B, Erman A, Rozen-Zvi B, Hirsh J et al (2008) Obesity-induced glomerular hyperfiltration: its involvement in the pathogenesis of tubular sodium reabsorption. Nephrol Dial Transplant 23:3946-3952

19. Chang AR, Wood C, Chu X et al (2020) Association of bariatric surgery with rates of kidney function decline using multiple filtration markers. JAMA Netw Open 3(9):e2014670

20. Chang AR, Chen Y, Still C et al (2016) Bariatric surgery is associated with improvement in kidney outcomes. Kidney Int 90(1):164-171

21. Navaneethan SD, Yehnert $H$ (2009) Bariatric surgery and progression of chronic kidney disease. Surg Obes Relat Dis 5:662-665

22. Kovesdy CP, Susan L, Zoccali C (2017) Obesity and kidney disease: hidden consequences of the epidemic. J Ren Nutr 27(2):75-77

23. Chen JH, Pan WH, Hsu CC et al (2013) Impact of obesity and hypertriglyceridemia on gout development with or without hyperuricemia: a prospective study. Arthritis Care Res 65(1):133-140

24. Hui M, Carr A, Cameron S et al (2017) The British Society for Rheumatology Guideline for the management of gout. Rheumatology. 56(7):1056-1059

25. Williams PT (2008) Effects of diet, physical activity and performance, and body weight on incident gout in ostensibly healthy, vigorously active men. Am J Clin Nutr 87(5):1480-1487

26. Kang EH, Lee EY, Lee YJ et al (2008) Clinical features and risk factors of postsurgical gout. Ann Rheum Dis 67(9):1271-1275

27. Friedman JE, Dallal RM, Lord JL (2008) Gouty attacks occur frequently in postoperative gastric bypass patients. Surg Obes Relat Dis 4(1):11-13

28. Raziel A (2018) Gout, obesity and bariatric surgery. Adv Obes Weight Manag Control 8(4):224-229

29. Dalbeth N, Chen P, White M et al (2014) Impact of bariatric surgery on serum urate targets in people with morbid obesity and diabetes: a prospective longitudinal study. Ann Rheum Dis 73(5):797-802

30. Sjostrom L, Narbro K, Sjostrom CD et al (2007) Effects of bariatric surgery on mortality in Swedish obese subjects. N Engl J Med 357(8):741-752

31. Nielsen SM, Bartels EM, Henriksen M et al (2017) Weight loss for overweight and obese individuals with gout: a systematic review of longitudinal studies. Ann Rheum Dis 76(11):1870-1882

32. Dalbeth N, Pool B, Yip S (2013) Effect of bariatric surgery on the inflammatory response to monosodium urate crystals: a prospective study. Ann Rheum Dis 72(9):1583-1584

33. Chen JH, Lee CH, Chang CM et al (2016) Successful management of newonset diabetes mellitus and obesity with the use of laparoscopic sleeve gastrectomy after kidney transplantation-a case report. Transplant Proc 48(3):938-939 
34. Schiavo L, Favrè G, Pilone V et al (2018) Low-purine diet is more effective than normal-purine diet in reducing the risk of gouty attacks after sleeve gastrectomy in patients suffering of gout before surgery: a retrospective study. Obes Surg 28(5):1263-1270

35. Romero-Talamás H, Daigle CR, Aminian A et al (2014) The effect of bariatric surgery on gout: a comparative study. Surg Obes Relat Dis 10(6):1161-1165

36. Zhang Q, Lou S, Meng Z et al (2011) Gender and age impacts on the correlations between hyperuricemia and metabolic syndrome in Chinese. Clin Rheumatol 30:777-787

37. Liu W, Zhang H, Han X, Zhang P, Mao Z (2019) Uric acid level changes after bariatric surgery in obese subjects with type 2 diabetes mellitus. Ann Transl Med 7(14):332

\section{Publisher's Note}

Springer Nature remains neutral with regard to jurisdictional claims in published maps and institutional affiliations.

\section{Submit your manuscript to a SpringerOpen ${ }^{\circ}$ journal and benefit from:}

- Convenient online submission

- Rigorous peer review

- Open access: articles freely available online

- High visibility within the field

- Retaining the copyright to your article

Submit your next manuscript at $\boldsymbol{\nabla}$ springeropen.com 